\title{
Mixed Reality-based Process Control of Automatic Printed Circuit Board Assembly Lines
}

\author{
Jürgen Hahn \\ University of Regensburg \\ Chair of Media Informatics \\ 93053, Regensburg, DE \\ juergen.hahn@ur.de

\section{Bernd Ludwig} \\ University of Regensburg \\ Chair of Information Science \\ 93053, Regensburg, DE \\ bernd.ludwig@ur.de
}

\author{
Christian Wolff \\ University of Regensburg \\ Chair of Media Informatics \\ 93053, Regensburg, DE \\ christian.wolff@ur.de
}

\begin{abstract}
A mixed reality (MR)-based concept for supporting and optimizing the way operators work with automatic printed circuit board (PCB) assembly lines, is proposed. In order to enhance the work process' interface, users are outfitted with a head-mounted display (HMD), so they can both actively access process relevant machine data and passively receive system notifications in a heads-up display (HUD), instead of having to manually query the terminal of the machine of interest at its very location. This approach was implemented and tested in a field study with one of the assembly lines of an electronics manufacturing services (EMS)-company. 30 staff members were recruited as test subjects and $90 \%$ of them appreciated the system deployment, due to its noticeable additional benefits compared to the status quo.
\end{abstract}

Permission to make digital or hard copies of part or all of this work for personal or classroom use is granted without fee provided that copies are not made or distributed for profit or commercial advantage and that copies bear this notice and the full citation on the first page. Copyrights for third-party components of this work must be honored. For all other uses, contact the Owner/Author.

CHI'18 Extended Abstracts, April 21-26, 2018, Montreal, QC, Canada C) 2018 Copyright is held by the owner/author(s).

ACM ISBN $978-1-4503-5621-3 / 18 / 04$

https://doi.org/10.1145/3170427.3188652

\section{Author Keywords \\ Assistance Systems; Mixed Reality; Industrial \\ Assembly; Human-Centered Computing}

\section{ACM Classification Keywords}

H.5.m. Information interfaces and presentation (e.g.

$\mathrm{HCI}$ ): Miscellaneous 


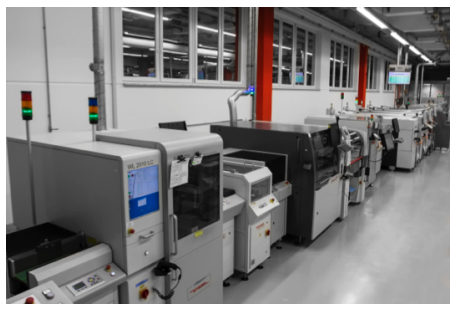

Figure 1: printed circuit board assembly line machine constellation, roughly 30 meters in length.

\section{Introduction}

Operators of industrial PCB automatic assembly lines, as shown in Figure 1, have to manage almost all of its machines in parallel. Their duties comprise three tasks, the most important of which is to ensure that the assembly machines never run out of components, followed by general surveillance or control of all line elements, in order to prevent or fix problems. Lastly, preliminary quality assurance (QA) is performed at random by manually inspecting whether the assembled PCBs are built according to plan. Operators rarely conduct major machine repairs themselves. The assembly line's machines feature decentralized active information access via their terminals and only passively alarm workers, once a production stopping error has occurred. Hence, users must patrol the assembly line in order to update their overview of each machine's state and to have a chance to prevent upcoming problems. This is especially the case when the worker has to restock a machine on one end of the assembly line and does not notice problems on the other end. Hence, this decentralization of information access creates productivity losses due to walking efforts, impediments of the worker's time management for routine subtasks and missing knowledge about machine states.

Therefore, we propose a MR-based system concept to centrally visualize the relevant assembly line live data in a HUD via a HMD. The software enhances the users work process, as demonstrated with a field study conducted in an industrial environment with productive live data.

\section{Related Work}

In previous work with a more technical focus, it has been shown that industrial workers definitely can benefit from head-worn assistance systems [2]. 30 test subjects were equipped with a HMD, displaying the retrieval locations and PCB installation locations of components, in order to train and guide the workers during the manual PCB assembly. In a field study, a day-to-day business assembly task unknown to workers was performed without error while using the system. Moreover, the software manages to strengthen the users' confidence in the correctness of his work.

Olwal, A., Gustafsson, J. and Lindfors, C. incorporated spatial AR on industrial CNC machines [5]. They display process data within the machine's safety glass, in order to create a physical relation between the data and its source. Only technical aspects are detailed in this paper and it remains unclear whether users benefit from this approach. However, the stereoscopic camera setup is quite bulky and therefore problematic when actually deployed in a live production setting as problems of work safety might arise. Our HMD approach might be preferable for an industrial context.

Fite-Georgel, P. argues whether there is reality in industrial AR [1]. In this paper, several AR projects are shown. According to the author, only $44 \%$ of all shown systems were developed with actual industry

cooperation, as well as user tests. The conclusion of the paper is that there is no real killer application, but many useful usage scenarios. The author confirms a reality in industrial $A R$, yet additional future work, as well as user tests, is an absolute necessity.

For related research on a conceptual level, there are two contributions influencing the proposed concept of this paper. In order to model our concept's assistance strategy, we have used the taxonomy proposed by Wandke, H. [8] and findings of Nitschke J.'s work [4]. The former illustrates a method to break down typical $\mathrm{HCI}$ assistance tasks into individual steps based on 


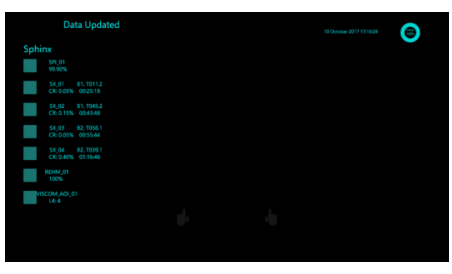

Figure 2: HUD in Overview Mode, showcasing a simple list of the machines currently tracked and their relevant process data (the data displayed is similar to the one in Figure 5, however only passively readable)

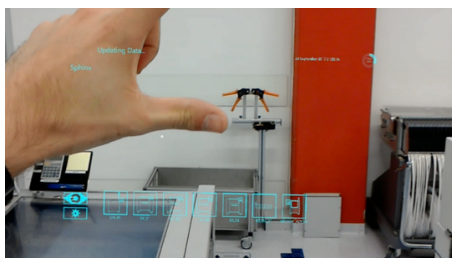

Figure 3: Ready Gesture with cursor

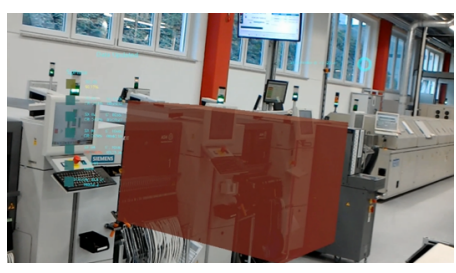

Figure 4: Alarm Highlighting of machine from further away

e.g., user goals, information reception or decisions concerning the choice of actions. The latter proposes that assistance bases on initiative and adaptability. Hence, each possible assistance type of afore mentioned taxonomy goes hand in hand with multiple dimensions of occurrences. The section about the way the proposed concept provides assistance to its user elaborates on these methods further.

\section{Concept}

The data which otherwise is scattered across all machines' terminals is condensed into one stream and is visualized in the central HUD of the HMD system. There, the worker actively or passively accesses the assembly line's process information. The concept is designed to adapt the three tasks of the work process, so the evaluation system features three modes of assistance. The first one being a passive information overview, consisting of a list of machines and their most important process data, as seen in Figure 2.

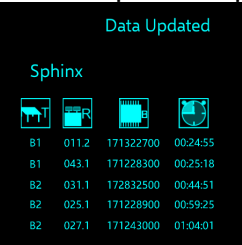

$0.05 \%$

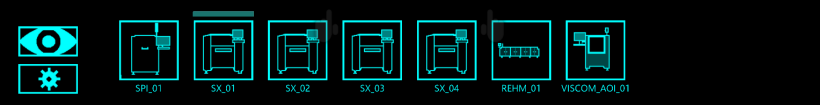

Figure 5: Active Manual Information Selection Mode (black background is meant to enhance readability; Figure $\mathbf{6}$ demonstrates the actual systems look during its use)
The second mode is split in two sub-modes. Figure $\mathbf{5}$ shows the state of the HUD when using the gesture interaction technique. After the system detects a hand with the HoloLens's Ready Gesture (Figure 3), the user can move a cursor with his hand. Then he can point and click (move index finger towards thumb) at one of the machines tracked in the bottom of the UI, in order to access this machine's data. Operators having to work with the machines stand next to them and look at them while at the same time they are able to access their process data, as shown in Figure 6. Here, the information is shown as a billboard, anchored above the machine. It is also completely encased in highlighting and the display color is determined by the machine's state: Problematic, yet acceptable states are in yellow while red depicts errors. The highlighting is always visible in these suboptimal states, regardless of user location, as seen in Figure 4.

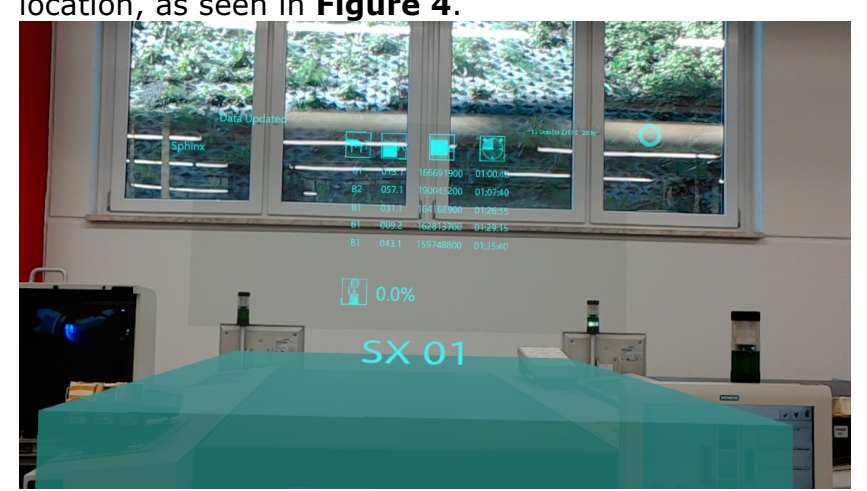

Figure 6: Positioning Context-based Selection Mode (the displayed data is perfectly readable in real use, yet screenshots captured by the HoloLens seem to make it appear otherwise)

The manual inspection mode is the third and final HUD mode, assisting the worker during the QA of the PCB assembly correctness. In order to activate this mode, 


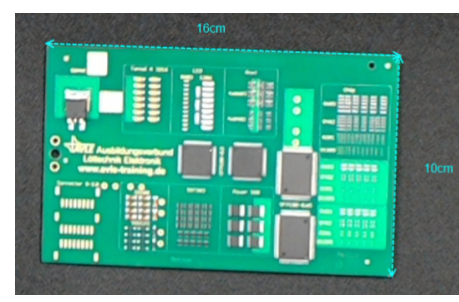

Figure 7: Trackless Trainings Printed Circuit Board exemplarily used for the system's manual inspection mode

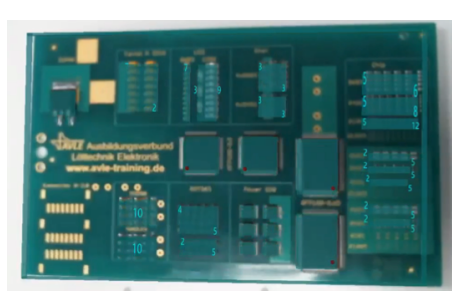

Figure 8: Overlay of the Holographic PCB Assembly Plan the user points and clicks on the eye symbol in the lower left corner of the UI (Figure 5). Once the user looks at a PCB like the one shown in Figure 7, the system overlays the real PCB with its holographic assembly plan, allowing the user to perform his task, as shown in Figure 8.

\section{Implementation}

The system is built for the Microsoft HoloLens [3] and uses the Unity Engine [7] as its application framework. The UI is completely kept in 3D and consists of fixed HUD elements like overview list and buttons as well as elements dependent on the context of positioning, like highlighting of machines or billboards. User location tracking is based on the use of the HoloLens's built-in sensors for room measurement. Hence, the system determines in which direction the user walks, looks and how much distance he or she covers in fractions of meters. With converting the production floor plan for use with the system, a virtual map is generated on software start up. However, an initial starting point has to be determined, requiring the user to stand at this specific location and look in a specific direction (calibration). The HoloLens' built-in sensors provide gesture recognition and the user's hand position is used in order to move the cursor and select UI items. The PCB detection for the manual inspection subtask is accomplished with a Unity plugin including the OpenCV library [6] for image processing functions. Thus, the PCB's pose towards the camera is calculated, as well as the coordinates of the holographic assembly plan overlay. The system downloads live data of the assembly line's machine via Wifi from the production server.

\section{User Assistance}

The system's assistance strategy model is based on the taxonomy proposed by Wandke, $\mathrm{H}$. [8]: For the three major work tasks the system provides display functionality and user information reception capabilities at a central HUD instead of via scattered terminals. According to Nitschke, J. [4], this is multidimensional in terms of system initiative and adaptability. Hence, the overview mode is the system's active method of assistance, because of autonomous updates, while the other modes passively serve the user, according to his input. Also, the system's assistance is adaptive, because changes in the assembly line context like critical error alerts trigger different assistance functionality. Visualizations of critical machine states and errors also provide activation assistance in terms of setting the user's goals. Here, coaching assistance is prevalent, because the users' motive of managing the assembly line is intensified. This is also true for the PCB QA task, because the operator immediately sees faults of the PCB assembly. In its two passive modes, the system provides assistance in terms of option supply, as well as filtering, because the user directly chooses which machine's relevant information is displayed in the HUD. In addition, the system provides indirect effect control in terms of feedback assistance. When the user fixes machine problems or restocks them, the software updates its displayed information after the task is completed and the new state is pushed to the system, allowing the user to verify whether his actions were successful or not.

The status quo also features assistance for the workers, but in a less efficient and effective way. To give an example, its activation assistance for alerts lack actual information about the problem, until the user reaches the terminal. An audible alarm and a blinking red 


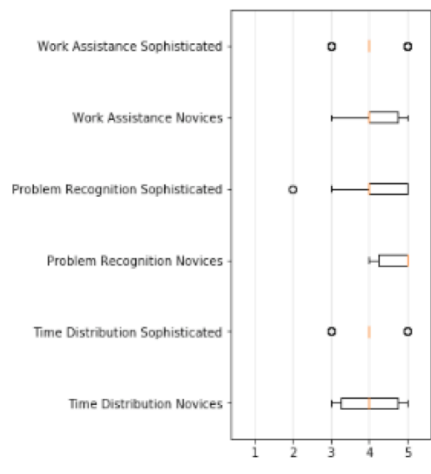

Figure 9: General Questionnaire Questions Results evaluated with a five-elements Likert scale ( 5 being the best possible tendency) in form of Boxplots with medians given in orange

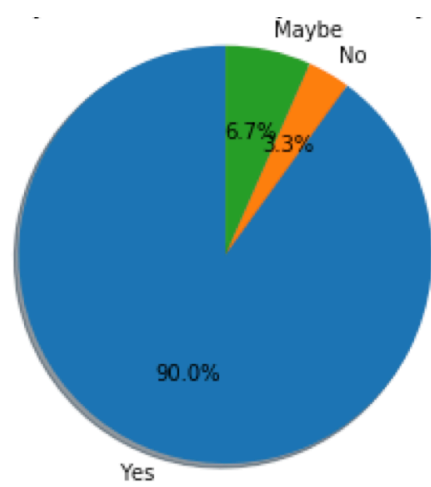

Figure 10: Approval of Deployment in live production warning light is all the assembly line-wide initial assistance provides, while critical machine states are only visible directly at the terminals in most cases. Those feature the information in an unprocessed way, making it hard for users to quickly find what they actually need to know and providing no coaching or filtering assistance.

Thus, our system prototype targets these work process deficits. By using the previously detailed assistance strategies, the system centralizes process information for the user to access from anywhere, instead of at specific locations only. When the user has to work at a specific machine, the data is displayed with physical context instead of a terminal, not distracting the user from the machine. The system allows the user to prevent errors, instead of only reacting to them, because it notifies him about deteriorating machine states. Because of that the operator is able to save redundant patrolling distances, as well as manage his external tasks like booking of order completion. The worker always knows the machines' states without having to worry about being surprised by a critical error. Longtime experts of the work process can operate assembly lines alone once equipped with the system. At the same time, novices benefit from the system, as it makes it easy for them to become accommodated with the workflow.

\section{Field Study}

The field study of the system, in order to test the proposed concept, was conducted in the automotive sector of an EMS company within a clean room production hall and was integrated into the live production. 30 test subjects ( 17 female and 13 male) were recruited among the department staff with an average age of 40 years (sample std. dev.: 10.42 years). 24 of them (11 female, 13 male) are dedicated assembly line operators with 6.5 years of work experience on average (std. dev. 4.7 years) and the others are process novices.

The field study was conducted within-subject and each test run took one hour to one and a half. The field test sequence consisted of device familiarization, working with the assembly line, filling out a questionnaire and a short interview. During the second phase, each test subject was instructed to restock an assembly machine, if this did not occur naturally during the live production, and perform the QA of the PCB assembly result.

The results of the study are given in Figure 9 and

Figure 10. The test subjects positively rated the effectiveness of the proposed assistance concept. Novices rated it slightly better than the sophisticated operators. Both types of workers claim that the work process is enhanced compared to the status quo (4 points median). In addition, they claim that they recognize problems faster and are better at managing their time.

The assistance during the QA of the PCB assembly result was also claimed to be sufficient, working sufficiently for bigger components and polarity, yet revealing weaknesses the smaller they get.

27 of the 30 test subjects also approve of a future deployment of the concept in form of a mature assistance system. Two of them are unsure and want to see further system iterations before committing to a final judgment. One test subject categorically refuses the use of such a system in general, seeing no need at all in changing the way production is currently performed.

However, this approval is bound to one condition, independent of the assistance concept: The hardware, in the users' eyes, has to improve significantly. This 
was the recurring theme during the experiment, as well as in the short post-test interviews. The subjects were generally pleased with the system, yet unhappy with the HoloLens device. Too bulky, heavy and partially neck straining were some of the attributes the test subjects brought forth. Hence, the conclusion of $90 \%$ of the test subjects was that the concept works well and noticeably enhances the work process regarding the assistance concept's goals. For actual deployment of an assistance system of that concept, the HMD has to meet ergonomic standards used in industry. It needs to be possible to wear the device for eight-hour shifts without suffering from side effects concerning users' health in the long term.

\section{Conclusion and Future Work}

With the results of the field study shown, the proposed concept is a sufficient enhancement for the operator's work process of automatic PCB assembly lines. In the future, system iterations of the concept, as well as refinements to the concept itself will be explored, once weaknesses are found in previous iterations. Long-term studies are also feasible, in order to get closer to providing assistance for a full shift, opposite to the onehour test sequence of this paper's evaluation.

Usability and user experience (UX) improvements or the proposal of guidelines for systems of this kind are areas for research as well.

With the hardware being the limiting factor, this paper's results point to future hardware developments. It would be feasible to apply the system's assistance design to ergonomically advanced technologies. In this way, a more immediate use of this paper's findings might be achieved.

\section{Acknowledgements}

The authors would like to thank Zollner Electronics $A G$, Zandt, Germany, for providing the project context of this paper, the required facilities for field-testing, as well as the test subjects for the study.

\section{References}

1. Pierre Fite-Georgel. 2011. Is there a reality in industrial augmented reality?. In $10^{\text {th }}$ IEEE International Symposium on Mixed and Augmented Reality (ISMAR), 201-210

2. Jürgen Hahn, Bernd Ludwig, Christian Wolff. 2015. Augmented reality-based training of the $P C B$ assembly process. In Proceedings of the $14^{\text {th }}$ International Conference on Mobile and Ubiquitous Multimedia, 395-399. ACM

3. Microsoft. 2015. HoloLens. Retrieved January 14, 2018, from https://www.microsoft.com/enus/hololens

4. Julia Nitschke. 2003. Assistenz bei Auswahlprozessen: Untersuchungen zu adaptiver und adaptierbarer Assistenz bei der Auswahl von Filmen, Ph.D. Dissertation, Logos-Verlag

5. Alex Olwal, Jonny Gustafsson, Christoffer Lindorfs. 2008. Spatial augmented reality on industrial CNC machines. In Proceedings of SPIE. Vol. 6804

6. OpenCV Team. 2017. Open Source Computer Vision Library. Retrieved January 14, 2018 from http://opencv.org/

7. Unity Technologies. 2017. Unity3D Engine. Retrieved January 14, 2018 from https://unity3d.com/

8. Hartmut Wandke. 2005. Assistance in humanmachine interaction: a conceptual framework and a proposal for a taxonomy, Theoretical Issues in Ergonomics Sciences, 6(2), 129-155 